\title{
Optimisation of multimedia over wireless IP links via $X$-layer design: an end-to-end transmission chain simulator
}

\author{
Catherine Lamy-Bergot • Roberta Fracchia • Matteo Mazzotti • Simone Moretti • \\ Esa Piri · Tiia Sutinen • Jing Zuo • Janne Vehkaperä • Gabor Feher • Gabor Jeney • \\ Gianmarco Panza $\cdot$ Peter Amon
}

(C) Springer Science+Business Media, LLC 2010

\begin{abstract}
End-to-end optimised Quality of Service (QoS) and its specific declination for multimedia applications with the end-user Perceived Quality of Service (PQoS) is nowadays a trendy topic in the literature. Many different techniques and approaches have been proposed, which are in general focusing on specific weak technical aspects of the transmission chain in the considered scenario. The end-toend optimisation of a complete system system is however more complex and its practical realisation remains to be achieved, especially with the added constraint to be transparently integrated in existing legacy systems so as to not perturbate their current modes of operation. In this paper, we propose an architecture set-up within
\end{abstract}

\footnotetext{
This work has been carried thanks to INFSO-ICT-214625 OPTIMIX project, which is partially funded by the European Commission within the EU 7th Framework Programme and Information Society Technologies.
}

C. Lamy-Bergot $(\bowtie) \cdot$ R. Fracchia

THALES Communications, Colombes, France

e-mail: catherine.lamy-bergot@fr.thalesgroup.com

M. Mazzotti · S. Moretti

CNIT, University of Bologna, Bologna, Italy

\section{J. Zuo}

University of Southampton, Southampton, UK

J. Vehkaperä · T. Sutinen · E. Piri

VTT Technical Research Centre of Finland, Oulu, Finland

G. Jeney · G. Feher

Budapest University of Technology and Economics, Budapest, Hungary

G. Panza

CEFRIEL/Politecnico Milano, Milan, Italy

P. Amon

Siemens Corporate Technology, Information and Communication, Munich, Germany 
the ICT FP7 OPTIMIX project to study innovative solutions enabling enhanced multimedia streaming in a point to multi-point context for an IP (Internet Protocol) based wireless heterogeneous system, based on cross layer adaptation of the whole transmission chain. The corresponding simulation chain architecture is detailed with the description of the existing and/or future features of each module.

Keywords End-to-end optimisation • High fidelity simulation • Quality of Service • Joint source channel (de)coding • Multimedia transmission •

Point to multi-point video delivery $\cdot$ Cross-layer design • IPv6 mobility •

Adaptive medium access control

\section{Introduction}

In wireless communications over power- and band-limited channels, telecommunications engineers face the difficulty of defining a suitable compromise for the contradictory requirements of large throughput, high robustness, low latency and low complexity. The minimum bit-rate at which distortion-less communication is possible is determined by the entropy of the multimedia source message. However, in practical terms the source rate corresponding to the entropy is only asymptotically achievable as the encoding memory length or delay tends to infinity. Any further compression is associated with information loss or coding distortion. An ideal and optimum source encoder generates a perfectly uncorrelated source-coded stream, where all the source redundancy has been removed; therefore, the encoded symbols are independent, and each one has the same significance. Having the same significance implies that the corruption of any of the source-encoded symbols results in identical source signal distortion over imperfect channels. Under these ideal conditions, according to Shannon's pioneering work [25], the best protection against transmission errors can be achieved even if source and channel coding are treated as separate entities. This milestone work, together with the obvious interest of designing separately source and channel standards explain why source and channel coding have historically been separately optimised.

However, as highlighted among others by Hagenauer [9], in practical situations the scenario is usually different. Mobile radio channels are very difficult channels in which many of the asymptotically valid hypothesis of Shannon's capacity theorem have limited applicability. Still, practical considerations on the difficulties to realise a joint design were dominating, in particular due to the necessity to take into consideration the impact of the network layer present in most communications nowadays. The usage of the (traditionally IP) network layer to simply interconnect wired and wireless equipments often led to further promote Shannon approach, by enforcing the impression that source and channel coding could not directly communicate and so could not be jointly optimised. Cross layer design [26] has been introduced to allow the evolution of the concept of joint source and channel coding, by targeting the joint design of the classically separated OSI (Open Systems Interconnection) layers, and, despite some cautiously pointed limits [13], the studies on this "protocol design done by actively exploiting the dependence between protocol layers to obtain performance gains" [26] has gained a large audience. While a large part of the literature is dedicated to the cross-layer for radio communications, solutions concerning the higher layers up to the application one $[8,16]$ have also been presented. 


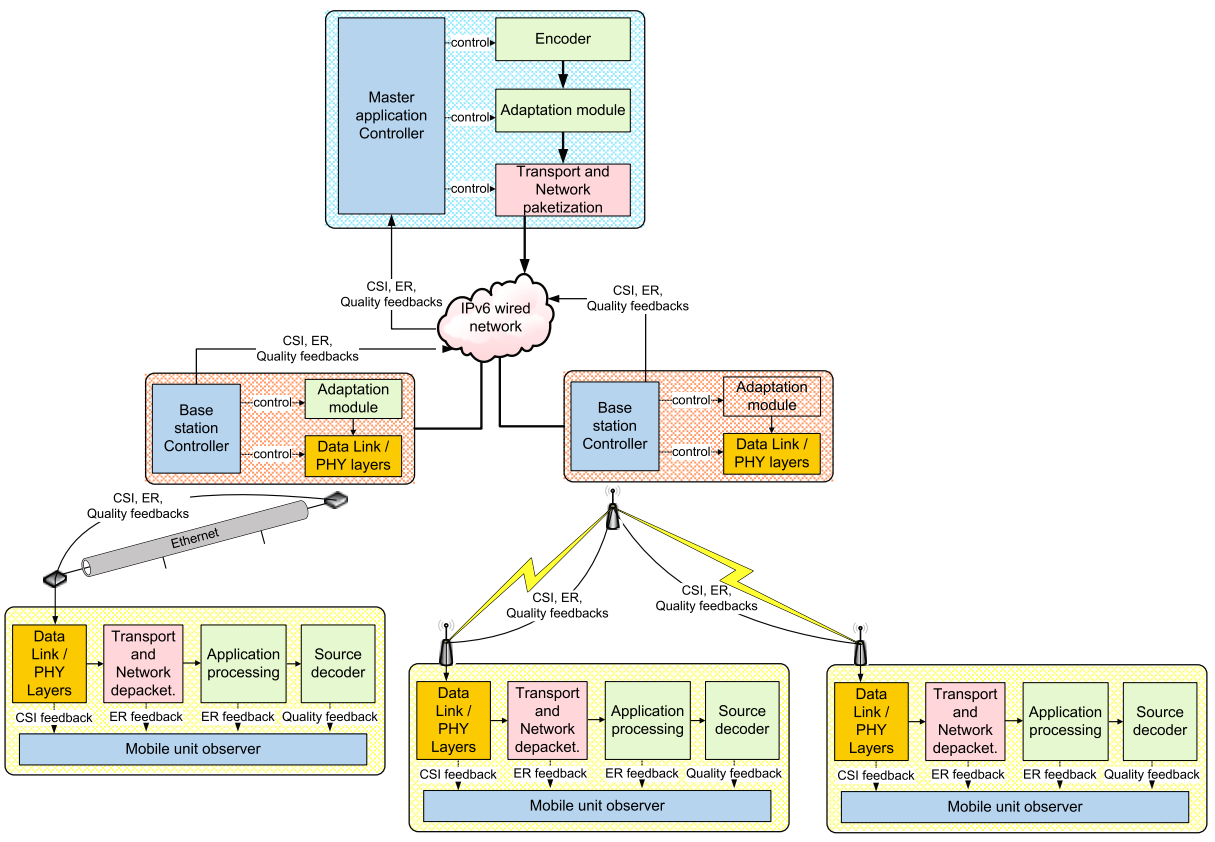

Fig. 1 OPTIMIX detailed functional architecture

In this paper, we present the approach followed by the ICT OPTIMIX project, that can be summarised as follows: "Controlling jointly the different modules of the transmission chain to optimise the communication". In order to meet this goal, we have defined a reference functional architecture, depicted in Fig. 1, aiming at modelling a point to multi-point multimedia transmission, from the source signal (audio or video) to the display for the end-user of the received and decoded signal, with in between standard and/or optimised representations of the different OSI layers of interest in this multimedia streaming context.

This document is organised as follows. Section 2 introduces related works, first on the end-to-end optimisation of a point to point or point to multi-points wireless transmission, and second on the simulation aspects themselves. Section 3 presents the OSI considered layers and the rationale on their use, while their implementation details are given in Section 4. Section 5 presents a set of results that have been obtained with the simulation framework and finally Section 6 draws out conclusions and future works.

\section{Related work}

In order to benefit from joint source channel coding/decoding (JSCC/D) and more generally cross-layer communications in real systems, control information need to be transferred through the network layers. This control information can for instance be Source Sensitivity Information (SSI), which allows to differentiate bits or packets 
according to a level of importance, or Channel State Information (CSI) which informs an emitter on the state of the channel it is going to use for its communication. This necessity of communication through layers usually impermeable to any crosslayer procedures did for long prevent many researchers to consider joint source and channel coding as a mature and realistic topic for wireless solutions. The time for cross-layer strategies has now come and the mechanisms for exchanging control information are being put in place. Among the first of these mechanisms, QoS oriented solutions offered at the network layer, namely differentiated services (DiffServ) and integrated services (IntServ), provide means for an application to have reserved transmission resources and specific service level from the interconnecting IP network. Another example of the inter-layer signalling can be found in the IEEE 802.11e standard where the QoS provisioning is achieved by coordination between the application and the medium access layers in wireless networks (WLANs). More complex solutions, that consider the whole protocol stack to perform a joint control from the application to the link layer, for instance for protecting video streaming over a wireless network, are also appearing. Typically, several solutions [2, 10, 18] propose the adaptation of the application, forward error correction (FEC) and/or medium access control (MAC) automatic repeat request (ARQ). Going beyond in terms of management solutions definition but also in implementation validation, different research projects such as ENTHRONE [27] or more recently SEA [30] have introduced respectively system management solutions or adaptation procedure to deal with end-to-end QoS improvement and wireless adaptation. These various solutions and many others in the literature are definitely proposing improvements and better quality of service for the end-user. The OPTIMIX project does not propose to replace them by introducing another management solution but rather allows the usage of our model in conjunction with most of these solutions, thanks to our approach transparent with respect to existing systems. This goal, as will be detailed in Section 3.4, is in particular enforced through the transmission of control information with a well-known existing framework, while rules for this control information are specific to OPTIMIX, and are defined by control algorithms attuned to the considered scenario.

With this goal, the need of a complex simulation system, offering the capability to jointly optimise several modules of the complex transmission scheme has emerged: only a full scale simulator can allow to envisage all possible interactions of the advances proposed by the research topics pursued within the OPTIMIX project at the different layers of the OSI protocol stack and at the same time to offer capability to test the compatibility of these new algorithms over existing protocols. As a consequence, the OPTIMIX partners have looked for a suitable environment of simulation. Different works, e.g. [12], have shown recently the number and variety of system simulators, as well as commented upon their evolution and growing usage. In our vision, the viability of such tools is dubious, when they are not enforced by a users community strong enough to maintain and let them coherently evolving with the research state-of-the-art, as was for instance proven by the YANS simulator [29]. Deciding to select among the most recognized and active system simulation systems, we have also elected to forego solutions with important license fees, to allow all partners of the project to share the same tool. Considering our need to model all OSI layers, and not "only" network ones, we looked among the system simulators and the $\mathrm{OMNeT}++[21]$ framework has attracted our attention. Having noticeably gained 
popularity in the last few years, mainly for the validation of networking solutions, $\mathrm{OMNeT}++$ is a tool developed to provide the fundamental features needed for the realisation of different simulation models. On the top of it, a variety of different models have emerged: e.g., the INET Framework, MiXiM [15] and the Mobility Framework (MF) [19], which proved the efficiency and the versatility of the whole framework. Nevertheless, we have also observed that the classical approach of OSI layers separate design still too often leads to define frameworks entering in deep details for given layers (of interest of the researchers developing them), while making over-simplifying assumptions for the other ones. In particular, the INET Framework which is a well-recognised networking model providing application, transport and network layer protocols description actually relies on a quite simple abstraction of the physical layer; conversely, rather accurate descriptions of the wired and wireless physical layers are provided by $\mathrm{MiXiM}$ and $\mathrm{MF}$, but their evolution is not necessarily immediate.

Considering here our will to build up an efficient cross-layer design for optimised video streaming, it appeared to us that building it with simplified models of upper or lower layers could only lead in practice to questionable results. Indeed, considering the high number of variable parameters we are proposing to study their mutual impact, from the physical layer (e.g. received power, number of interfering signals, multipaths, ... ) to the application level (e.g. taking into account a real source and not a traffic generator) and including also signalling possibilities (e.g. different overhead possibilities based on the various standard implementation of RTP (Realtime Transport Protocol), transport and IP layers, aggregation of multiple receivers feedbacks, ...), obtaining reliable simulations with simplified models in different layers seems dubious. Furthermore, one of OPTIMIX goals, in order to back up the results obtained with the simulator framework, is to build a reduced-size demonstrator enabling to validate "in real life" the results obtained with the simulator. As a consequence, we chose to develop our own OMNeT ++ framework for high fidelity simulation, with for many classes and modules a full $\mathrm{C} / \mathrm{C}++$ implementation of the algorithm or protocol, that will be later used for the demonstrator. This approach, which builds upon pre-existing $\mathrm{C} / \mathrm{C}++$ implementation of several features, is a guaranty for us to obtain a fine model of the different considered layers, thus satisfying our needs and wishes.

\section{The OPTIMIX architecture}

The joint end-to-end optimisation approach of the OPTIMIX solution is based on the enhancements of many different OSI layers and on the introduction of controlling modules that will ensure the jointly optimised use of the different protocols to maximise the quality perceived by the end-user. As shown in Fig. 1, the protocol stack has been separated into three main groups, that are the application and session layers (represented in green colour, and further detailed in Section 3.1), the transport and network layers (represented in pink colour, and presented in Section 3.2) and finally the radio access (data link and physical layers, represented in orange colour, and described in Section 3.3). The specific controlling framework, with the units deciding of the strategies to implement and the measurements units are drawn in blue colour, and their mode of operation is explained in Section 3.4. 


\subsection{Application and session}

At the application layer, two video coding schemes-H.264 Advanced Video Coding (AVC) and Scalable Video Coding (SVC) - and two audio coding schemes-AAC+ (evolution of Advanced Audio Coding) and AMR-WB+ (evolution of Adaptive Multi Rate-Wide Band) - are considered in the OPTIMIX context. For both applications, the well known Real Time Streaming Protocol (RTSP) [24] and the RTP Control Protocol (RTPC) [6] are used for session initiation and maintenance. Audio/Video synchronization aspects are also foreseen, and will be handled thanks to a shared RTP timestamp information.

H.264/AVC, standardized jointly by the ISO/IEC MPEG and the ITU-T VCEG groups, represents the state-of-the-art in single layer video coding, and is therefore a reference for our system, to which additional features compared to the original reference software package [11] have been introduced by the project: e.g., enhanced capabilities to operate in an error prone environment (i.e., packet loss and bit errors), soft-input decoding of variable length codes to deal with bit errors, and better error concealment techniques to handle unavailable information due to packet loss. H.264/SVC has been standardized only recently as an annex to H.264/AVC. It introduces enhancement layers to a base layer stream compatible with H.264/AVC. In addition, temporal scalability can be introduced by using prediction structures allowing for the removal of intermediate frames. As for H.264/AVC codec, additional error resilience techniques [28] have been introduced in the OPTIMIX decoder.

Finally, another optional functionality has been added in the so-called application processing sub-layer, which allows to perform joint source/application error correction optimisation when operating over pre-coded streams, by typically adding an application-layer forward error correction code and/or transcoding the original data stream. In one mode of realisation, the error correction code is integrated directly into the data stream, via a plug-in module to a standard codec. Similar adaptation modules have also been inserted in the middle of the transmission chain, for instance at base stations, to allow adaptation (stream decimation, insertion of further error correction, decode and forward strategies, ...) when needed in the transmission process.

\subsection{Transport and network}

The transport and network part regroups several sub-layers, among which we find first the RTP protocol [6] one, which provides end-to-end network transport functions suitable for networked transmission of real-time data such as video or audio. Integrated in the OPTIMIX communication system and used in combination with the transport protocol for synchronization between audio and video, the RTP module supports generic, H.264/AVC and H.264/SVC payloads. Moreover, specific extensions in the RTP FEC approach with two different FEC modes have been introduced to cope with possibly remaining bit errors. The first mode is a systematic one (fully transparent for users unaware of the FEC feature), where information bits are kept untouched and redundancy packets are added. This method is based on ReedSalomon codes and defines a new payload type for redundancy packets. The second mode is non-systematic (information bits are used to generate a non-systematic 
payload): information bits are no longer intact and only redundancy packets are transmitted. This method is based on rate compatible punctured convolutional (RCPC) codes with four different classes of protection : i) unique protection (EEP) with definition of (size, rate) in the extension; ii) unique class except for 40 protected bits (Network Abstraction Layer (NAL) header+1st byte) to the maximum rate; iii) two classes, with no default overprotected bytes, with definition of (size, rate) in extension; iv) three classes, with no default overprotected bytes, with definition of (size, rate) in extension. It has to be noticed that in all cases, the output of the RTP module is standard RTP packets, even when FEC is used. Parameters of each correction mode will be evaluated and selected by simulation. Below the RTP module we introduce the Secure Real-Time Transport Protocol (SRTP) [3], which optionally provides content security for the audio/video transmission. When using SRTP, the content is secured, thus only the dedicated receivers are able to decode it: the integrity is offered by ensuring that the received stream is really coming from the authenticated server and has not been modified during the transit. SRTP is an Internet Engineering Task Force (IETF) standard with default ciphers and integrity protection algorithms, which adds reasonable overhead to the stream size and can be tailored to the application needs. In the OPTIMIX communication system we are considering to use different algorithms to achieve better performance. First, to increase the ciphering speed, the classical Advanced encryption Standard (AES) cipher is replaced by a stream cipher, called ChaCha [4] which is a product of a recent EU-IST research project on ciphers that can over-perform the state-of-the-art AES. Second, due to the wireless nature of the transmission, as bit errors are common, we are working on replacing the authentication algorithm to avoid a systematic discarding of frames with a few bit errors eventually caused by the wireless channel. Current work is on-going on the selection of an approximate authentication algorithm, able to authenticate (albeit with less guarantees than a classical authentication algorithm) the stream regardless of small, natural transmission errors.

The transport protocols selected for OPTIMIX multimedia transmissions are the classical User Datagram Protocol (UDP) [23] or its derivation UDP-Lite and the more recent Data Congestion Control Protocol (DCCP) [14], which interests us due to its congestion control mechanism and the absence of retransmission procedures. This last feature, which usually introduce long and useless delays for video streaming, make of DCCP a particularly well-suited protocol to the scenarios under consideration. It should be pointed out that for validation purposes, the possible usage of the Transmission Control Protocol (TCP) is also being considered. As a feature extension for transport protocols (UDP, DCCP) do not providing retransmissions, we have also introduced Packet Erasure Correcting Codes (PECC) techniques based on properly designed Low Density Parity-Check (LDPC) codes. The structure and management of data and parity packets is based on the MBMS standard [1]. Packet erasure codes are proven to be very effective in delay-sensitive point-to-multipoint communications and, allegedly, they will be very common in the near future. The introduction of our LDPC-based solution in real communication systems would not require big investments by service providers, since it is completely software-implemented and patent-free. It is foreseen to evaluate with the OPTIMIX simulation the respective interest, from the performance and deployability point of view of both RTP FEC and PECC approaches to correct any remaining errors or losses caused by the end-to-end transmission and left uncorrected by the PHY 
layer FEC. It should be noted that their use could also be eventually combined, with having RTP FEC cope with errors and let PECC deal with the packet losses. As a result of the MBMS-compliant FEC encoding process, four bytes (denoting the Source Block index (ID) and the Encoding Symbol ID) are appended to the payload of each transport layer source packet and several redundancy packets are generated and passed to the lower layers for transmission. In particular, the payload of each redundancy packet is constituted by one or more parity symbols, in addition to 7 bytes carrying information necessary for a correct decoding (Source Block ID, Encoding Symbol ID, number of source block rows and applied code rate).

Below the transport protocol we introduce the Host Identity Protocol (HIP) [20] sub-layer which is used to provide advanced mobility mechanisms and perapplication mobility (every application being able to have different policies for handover management). The network layer found hereafter is based on the use of the IPv6 protocol [7] together with its advanced built-in features. For example, IPv6 anycasting allows the transmission of packets to one of the hosts belonging to a specified group, used for feedback aggregation in the OPTIMIX system. Indeed, all the hosts, which receive the video stream, send feedback information to a specific anycast address; the network forwards these packets to the closest host which has the anycast address (i.e., the streaming server and the aggregation servers). In the worst case, where anycast is of no use, all feedback packets are directly forwarded to the streaming server directly. If one of the aggregation servers is along the path back to the streaming server, and the network supports anycast based routing, the feedback information will arrive at the aggregation server. The aggregation server can combine the different feedback messages thus allowing one single stream from the aggregation server to the streaming server. This solution saves bandwidth on the network and provides saving in the number of connections which must be maintained by the streaming server.

\subsection{Radio access}

In order to reduce the bandwidth occupancy of multimedia streaming applications and signalling, which is a serious issue over IP wireless networks, header compression is introduced in the OPTIMIX system. It has to be noticed that ROHC (Robust Header Compression) [5] utilisation is expected to be particularly useful in our proposed system, where the use of UDP-Lite or DCCP is combined to RTP packetisation and many feedbacks are transmitted from the clients to the server.

Starting from the ROHC standard for IP, UDP, RTP and TCP, OPTIMIX is developing a new header compression function with the following features: $\mathrm{i}$ ) capability to compress the header introduced by the DCCP transport protocol which, being up to twice longer than the UDP header and introducing several packet formats, requires particular attention; ii) compression adaptation to transmission quality, whose information is collected by the mobile observer and received via the application controller; iii) compression of multicast transmissions and adaptation of the compression profiles to the needs of different users.

At the data link layer (DLL) and at the physical layer, multiple transmission schemes are considered, i.e. the main elements included in the IEEE 802.11g specifications and a more complex multiple-input multiple output (MIMO) Orthogonal Frequency Division Multiple Access (OFDMA)-based architecture. The 
reason why we do not take into account a single downlink architecture is twofold. First, the end-to-end benefits of the proposed cross-layer approach are not strictly dependent on a given radio transmission scheme. Thus, having the possibility to test our solutions with different access schemes, channel codes, modulation techniques and multi-antenna architectures is of fundamental importance. Second, adaptive and opportunistic cross-layer algorithms for user scheduling and resource allocation provide the most interesting gains over traditional radio access schemes when multiple degrees of freedom are present, in terms of time slots, sub-carriers and spatial dimensions to manage. The possibility to combine different multiple access schemes, like Time Division Multiple Access (TDMA), OFDMA or Space Division Multiple Access (SDMA), is a key element which determines substantial growth in the multi-user throughput for recent and future radio standard (e.g. WiMAX, 3GPP LTE, ...).

\subsection{Controllers and observers}

Different entities are involved in the JSCC/D adaptation process proposed by OPTIMIX, namely the Application Controller, the Base Station (BS) Controller and the Mobile Observers. This approach comes from the splitting of the general joint adaptation problem into a number of sub-problems, addressed by distinct controlling modules strictly cooperating in order to optimise the end-to-end perceived quality. This collaboration between the application controller and the base station controller is realised through a cross-layer exchange of side information and control signals across the network (provided by the observers).

The application controller can be seen as an intelligent streaming pump implementing the controlling strategies ensuring that the compression and protection functions are decided jointly and efficiently from the end-user point of view. This intelligence in the streaming server is driven by a controller whose role is to improve the long term average received video quality, by controlling the compression and protection levels as well as the different modules in the transmission chain and adapt their parameters based on the feedback information it receives on transmission conditions. In that aspect, the application controller is the master of the whole system, as it transmits QoS targets to the different base station controllers, which will make their best to meet such targets, and inform the master of their success, or failures, to decide of new parameters based also on the observers feedbacks.

The Base Station Controller is designed to manage the large set of degrees of freedom that the radio resources typically provide in point-to-multipoint communication scenarios, in terms of frequency, time and space multi-user allocations. Best-effort adaptive algorithms for the BS Controller have been considered within the project, capable to intelligently (and fairly) serving the users according to their priorities and their radio channel state. One of the main goals of the BS Controller is to exploit the flexibility of different channel coding and modulation techniques in order to jointly adapt the wireless transmission scheme to both the source characteristics and QoS rules specified by the Application Controller and to the radio channel state experienced by the different users. In fact, a detailed set of CSI is supposed available at the base station, mainly through feedback channels from the receivers, and proper SSI information come from the upper layers, together with a set of requirements and constraints imposed by the Application Controller. 
The iterative exchange of information relevant to requirements and feedbacks between adaptive entities and the techniques capable to exploit them constitute the core of our preliminary design of the JSCC/D Controllers. In particular the Mobile Unit Observer is another key-element of our approach, since its main purpose is to provide to all the requiring entities the needed feedback information from the end-user mobile terminal. The Observer runs functions of a triggering engine (TRG) which provides a unified service for cross-layer information collection, temporary storage, and dissemination within the network protocol stack. The TRG was originally developed in the FP6 IST-Ambient Networks project to assist handover operations, and has been extended and adapted in OPTIMIX simulator for feedback information signalling between the system elements. TRG is the central component of the cross-layer signalling architecture based on the triggering framework [17]. The role of TRG in the triggering architecture is to collect information (triggers) of several events occurring at different layers of the protocol stack, to process the information carried in the events into triggers, and to deliver the triggers to their consumers according to specific rules and policies defined for the trigger delivery. For example, consider the case where a video quality estimation is performed after the decoding process. This event constitutes a Quality Information trigger collected by TRG, which, in turn, delivers the trigger to the Master Application Controller which has subscribed for this trigger.

Triggers sent by the different observers can then be aggregated in the networks using the IPv6 anycast functionalities, as described in Section 3.2.

\section{OPTIMIX simulator implementation}

As stated in Section 2, to validate our architecture we have selected a generic framework built on the OMNeT ++ simulation tool and we use as far as possible only the $\mathrm{OMNeT}++$ most generic features (e.g. discrete event scheduling and simple and easily re-usable $\mathrm{C} / \mathrm{C}++$ code implementation) in order to offer for each function and layer the degree of refinement needed for our overall end-to-end optimisation. Making this choice to ensure that our framework would completely fit our purpose, i.e. the establishment of a generic architecture to simulate transmission of multimedia content over hybrid wired/wireless networks, we have accepted to pay the price of a more lengthy implementation task, which we hope will be valuable for the build-up of the OPTIMIX demonstrator, in which we plan to re-use the $\mathrm{C} / \mathrm{C}++$ algorithms for OPTIMIX specific functions.

Following the architecture presented in Fig. 1, the corresponding simulation implementation has been defined, as detailed in Fig. 2. In this second figure we show the simulation architecture itself, with the different elements implemented within the generic OMNeT++ framework, and with the same colour codes as in Fig. 1.

For each module of the simulator, the communication is foreseen on two plans, corresponding to the traditional approach of data/signalling separation, which is being especially enforced in OPTIMIX system due to the large importance devoted to the signalling in the controller/observer work. To achieve this goal, the complete protocol stack from the application to the physical layer is simulated, and the real bits and bytes of the messages transferred on the radio channel are exchanged. 


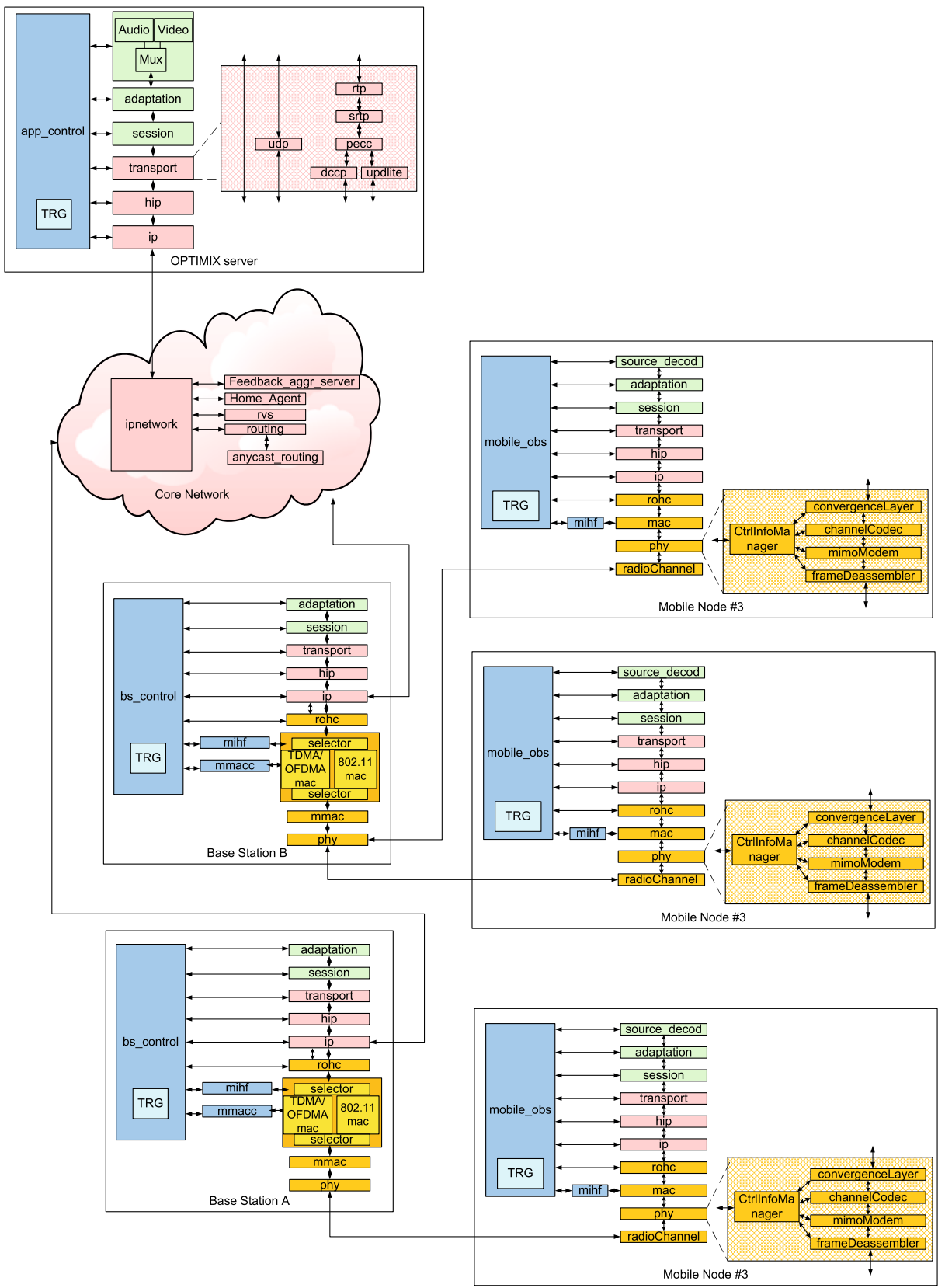

Fig. 2 Overview of the OPTIMIX OMNeT ++ complete simulator (realisation case with two base stations and three mobile nodes)

Exploiting the OMNeT ++ framework, the communication between the different modules and nodes of the system is performed by means of messages which, as much as possible, use as reference format the standard ones. Typically, for data, the IETF 
datagrams (IPv6, DCCP, UDP-Lite, RTP,...) for upper layers and standard radio packets for lower layers are considered, while for signalling, existing specifications (e.g. RTSP, RTP Control Protocol (RTCP), Internet Control Message Protocol $(\mathrm{ICMP}), . .$.$) are studied solutions.$

\subsection{Application and session}

At the application layer, as described in Section 3.1 the source coding module receives a request for a specific content driven by a RTSP client-server approach. The transmission is thus characterized by session initiation and session maintenance phases. On the one hand, a link (called session) between the server and the terminal for the exchange of the parameters of the audio and video streams is set up and destroyed. The link set-up, the parameter exchange, and the link destruction are achieved by RTSP handshaking that consists of standard DESCRIBE, SETUP, PLAY, and TEARDOWN messages. On the other hand, the established session has to be kept up and running while transmission is in progress. Session maintenance is carried out by periodically transmitting RTP control protocol messages between the server and terminal session layers. RTCP protocol is not only used as a means for session maintenance, but it is also used for supplying the terminal side with synchronization information, and for monitoring the status of the communication link.

The requested content may be either in raw YUV colour space 4:2:0 format video data, to be encoded within the simulation, or pre-coded H.264 video data. The first approach is used especially for H.264/AVC, since the data-rate can be adapted after the encoding step with more difficulties. The alternative approach with a pre-coded video file is considered valid for SVC, where the data-rate (and also the image size and frame rate) can be adapted in the compressed domain by simply removing packets (i.e., NAL units) from the bit-stream. The raw video data is encoded or the pre-coded file is selected following the indications provided by the application controller.

The H264/AVC module implements all the features described in the previous section. For the H264/SVC module the settings are as follows: i) Scalable Baseline profile; ii) Spatial scalability with 2 layers; iii) Dyadic temporal scalability with a group of picture (GOP) size of 8 frames (optionally 16) and an Intra period of 32 frames (optionally 64 or 128) at the highest spatial layer, a low delay configuration with hierarchical P frames only (no B frames); iv) Application of SNR (Signal to Noise Ratio) scalability open. A robust SVC decoder with different error concealment algorithms [28] has also been implemented providing interpolation (by using correctly received information inside the same frame), frame copy (by utilising temporal macro block copy from earlier frames), utilisation of spatial enhancement layers when the base layer is missing, up scaling lower SVC layer in case of missing higher spatial enhancement layer.

At the receiver side, the received encoded stream is thus decoded and displayed. In the first simulator version, audio codecs are not present, but they will be introduced in the next release of the simulator. 
4.2 Transport, network and packetisation

The transport layer module implements various transport protocols. UDP-Lite and DCCP are used for the transport of real-time data and their impact on the video quality will be compared. Over these transport protocols, the RTP protocol is considered, with optionally its specific secure profile that provides ciphering and authentication in unicast and multicast modes. Both RTP and RTSP implementations include the new features described in the OPTIMIX system specifications in Section 3.2.

Control messages, usually transmitted in real life situations by reliable transport protocols as TCP, are in OPTIMIX transmitted using UDP, since TCP retransmissions are expected to be useless due to the inevitable introduced delay.

The advanced mechanisms introduced and designed to allow users mobility and based on the Host Identity Protocol (HIP) are currently only partially implemented, as this is an extremely challenging task. Nevertheless, the simulation will contain HIP support: basic/limited support will be provided which is enough to demonstrate the effect of mobility. For comparison purposes, the usage of the HIP module can be rendered optional.

The IP layer module in the simulation chain encapsulates downstream packets received from the transport layer into IPv6 packets and, in the opposite direction, decapsulates IPv6 packets and sends them to the appropriate transport layer module.

The impact of an IPv6 wired network, corresponding to a local network or to Public Internet crossing is modelled with a network module. The purpose of this module is to represent in a scalable manner the wired trunk of the telecommunication infrastructure, by introducing the effects on the data transmission (packet loss and delay) due to the crossing of multiple IP routers. The loss and delay parameters are established via analytical modelling based on statistical distributions resulting from the analysis of measurements taken in the real world environment (i.e. the Public Internet). The type and shape of such distributions match the histograms of the loss and delay of packets in core IP networks of the Public Internet. The first order statistical values of the employed distributions (mean, variance, ...) can be configured, according to the number of routers meant to be crossed and the imposed average values of loss and delay at each router interface. Such average values can be set referring to a specific network, with or without QoS support. The point to multipoint transmission with multicast functionality is emulated by a routing function inside the IP Network Module that realises the replication of the data flow addressed to the different users when needed.

\subsection{Radio access}

The header compression module introduced in the simulator receives network layer packets and produces packets with a compressed header. It is integrated in the simulator above the MAC layer in both base station and mobile users. This module, in addition to the RTP/UDP/IP header compression specified in the standard, will implement the header compression for DCCP packets. The three operation modes (Bidirectional Reliable Mode, Bidirectional Optimistic Mode, Unidirectional Mode) of ROHC are developed. 
As already stated in Section 3.3, a data link layer implementation is technologyspecific, so we decided to implement different solutions in our simulator. Basic DLL services specified in two standards of interest for OPTIMIX (i.e., IEEE 802.11g and IEEE 802.16e) are introduced, with amendments to allow end-to-end optimisation. One of the key additional techniques is a partial checksum, using only DLL headers in the checksum calculation, which allows to pass a corrupted payload data to higher layers for further processing. In the future version of the simulator, a support for prioritization using buffers above the actual DLL will be added. The buffers are used to prioritize video packets (e.g. layers of SVC video) in the access point destined to the different users.

The physical layer (PHY) module receives data packets from the DLL and arranges them for the transmission over the radio channel. At the receiver side, it has the dual role of demodulating and decoding the received symbols before passing the correspondent packets to the upper layers. The PHY module is composed of several sub modules, i.e. channel co-decoder, MIMO-OFDM mo/demodulator and physical frame de-assembler unit. The possibility to choose between various channel coding and MIMO-OFDM modulation schemes permits to evaluate the results of the end-to-end optimisation process addressed in the project in case of different radio communication scenarios, e.g. WiMAX or $802.11 \mathrm{a} / \mathrm{g} / \mathrm{n}$. Currently, the channel co-decoder includes RCPC codes and Rate Compatible Punctured Irregular Repeat Accumulated (IRA) LDPC codes. Orthogonal Frequency Division Multiplexing (OFDM) modulation is supported, as well as multiple transmitting and receiving antennas. More complex space-time coding (STC) and linear beam forming techniques are also implemented.

Finally, the transmission is done over a frequency-selective channel sub-module which introduces the typical radio transmission impairments met in wideband mobile communications (e.g. different Rayleigh fading for the various sub-carriers, lognormal slow fading and additive thermal noise).

\subsection{Controllers and observers}

The application controller, using as input encoding configuration and wireless network conditions, selects a set of encoding parameters which are then transmitted to the encoder module where they are used or to encode a raw stream in function of the user needs or to select the most suited among pre-coded streams. If applicable, the controller will also decide on the level of protection introduced in the higher layers (with application processing, RTP FEC or PECC modules). For multicast video transmissions, the encoding parameters should be selected taking into account different feedbacks received by different users. How to weight the different information which will be subject of further studies.

The BS Controller module implements algorithms to schedule multi-user transmissions, to allocate radio resources and to adaptively select coding and modulation parameters. In particular, it is based on low-complexity techniques based on stochastic Lagrangian dual-function optimisation, e.g. aimed at reaching sum-rate (SR) or weighted sum-rate (WSR) maximizaton.

The Mobile Observer implementation follows the description in Section 3 and it is based on the triggering framework. The Mobile Observer includes the TRG functionality so it is responsible for passing feedback information between different 
sources and consumers. This means that the Mobile Observer does not measure and calculate statistics from each layer or module; it is just a delivery module for crosslayer information within a network entity or between network entities. Each module producing cross-layer information (i.e. a trigger source) generates the trigger by itself with a unique index and type and passes it to the Mobile Observer. The Mobile Observers takes care of passing the trigger to the consumer which has subscribed to this specific trigger. In the Mobile Observer, the TRG implementation is coupled with the IEEE 802.21 implementation. The Media Independent Handover Function (MIHF) module provides a few of the main services specified in the IEEE 802.21 standard, and TRG allows expanding the IEEE 802.21 event reporting to a signalling beyond the scope of IEEE 802.21, that is, to full cross-layer and end-to-end scope. MIHF provides events related to current link conditions to MIH User (MIHU), which is a sub module inside the Mobile Observer module. These events are initiated by the MAC layer and include timely values for several MAC and PHY parameters. MIHU, in addition to its internal decisions on improving link efficiency according to the parameter values, also converts the IEEE 802.21 events to TRG triggers. For example, each parameter in the MIH_Link_Parameters event is converted to a trigger with the pre-specified ID and type for the identification of the parameter. Thus, not only MIHU can utilize the parameter values included in the IEEE 802.21 events, but also each layer or module using the services of the triggering framework through the trigger subscriptions. MIHF on MSs and BSs can register with each other in order to be able to subscribe and receive remote events in a fast and lightweight way over the Layer-2 communication. Also possibility to use IEEE 802.21 command service is considered in the implementation to command the MAC layer behaviour.

\section{Interest of the simulation and results}

Aiming at proposing solutions suitable for the next generation of networked radio communications, and willing to take into account as many options as possible, it is clear complete combination of all possible parameters realisations can not be systematically tested. This is why, as with any complex simulation, the results will be particularly of interest when a detailed (and possibly realistic) scenario is considered and compared with recent or legacy standard solutions, to obtain fair comparison and gains figures. Nevertheless, another usage of the simulator is to allow the validation of specific modules and algorithms, and their performance evaluation when applied in a complete and realistic end-to-end transmission context, i.e. when taking into account the possible impact of said considered modules or algorithms in a high quality simulation of radio channel, PHY layer, wired network, .... Examples of such results obtained with the simulator are presented in the following. Due to the complexity of the simulator, only a handful of results are here presented, however it should be noted that the simulator [22] will be released by the end of OPTIMIX projet for further usage in the scientific community.

In Fig. 3 we show the performance of a couple of the implemented modulation schemes. Figure 3 compares the binary error rate (BER) performance of Phase Shift Keying (PSK)/Quadrature amplitude modulation (QAM) schemes and SpherePacking based Space-Time Block Coding (SP-STBC) schemes when communicating over correlated Rayleigh fading channels having a normalized Doppler frequency of 

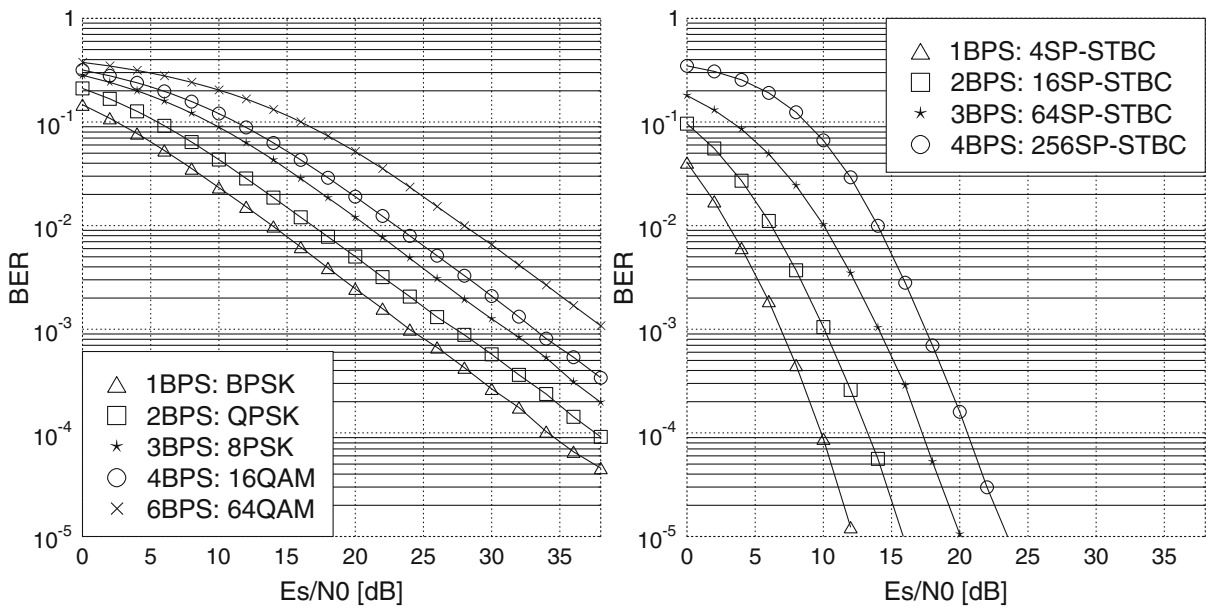

Fig. 3 Comparison of the BER simulation performance when communicating over correlated Rayleigh fading channels

0.1. More specifically, each of the PSK/QAM scheme employs one transmitter and one receiver while each of the SP-STBC scheme invokes two transmitters and two receivers. As can be seen from Fig. 3, the 4SP-STBC scheme outperforms the BPSK scheme by approximately $17 \mathrm{dBs}$ at $\mathrm{BER}=10^{-3}$, when aiming for a throughput of 1BPS (bit per symbol). Similarly, all other SP-STBC schemes outperform the PSK/QAM counterparts significantly when aiming for a throughput of 2BPS, 3BPS and 4BPS.

In Fig. 4 we report the evaluation of the performance achieved in case of the header compression, over a correlated Rayleigh fading channel as with the BER performance curves. For sake of simplicity, we report here the results obtained with the simplest case of BER simulations (corresponding to a BPSK modulation and no channel coding used for the transmission). Using the signal-to-noise ratio $E_{S} / N_{0}$

Fig. 4 ROHC performance evaluation over a correlated Rayleigh fading channel

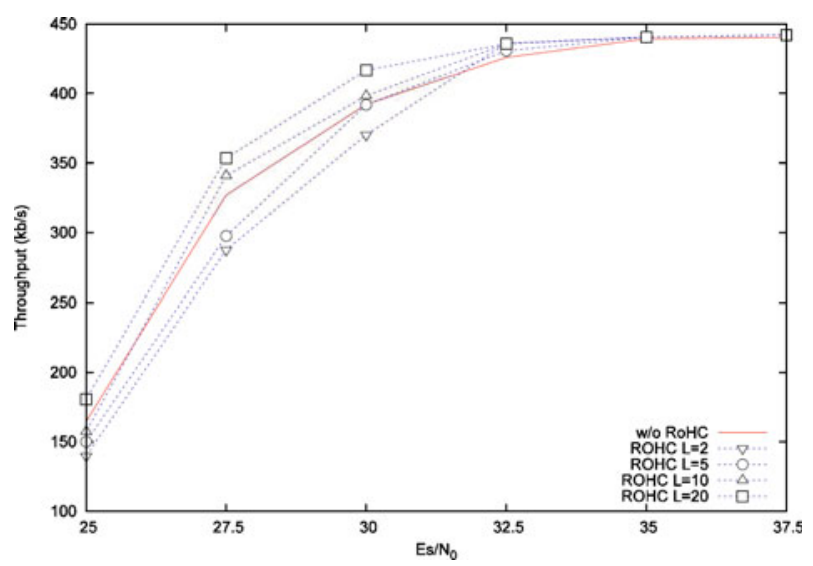


as abscissa as in Fig. 3, Fig. 4 reports the throughput achieved at the transport layer for different signal-to-noise ratio values for different values of the ROHC algorithm $\mathrm{L}$ parameter in unidirectional (U mode) mode, which defines the stability in a compression state. In practice, for high values of $\mathrm{L}$ the compressor stays longer in the less compressed states, which thus results in a bigger average header size while but also a lower probability of context errors. As there is no error correction coding on the PHY layer in this simulation case, we report only values of $E_{s} / N_{0}$ between 30 $\mathrm{dB}$ and $40 \mathrm{~dB}$ since lower values would generate important BER and consequently a very low perceived quality. We can observe that while for low error probabilities (e.g., for $E_{s} / N_{0}=32.5 \mathrm{~dB}$ ) performance obtained with header compression is better for any compression parameters, the trend significantly changes when the error rate increases. As a matter of fact, the $\mathrm{L}$ parameter has to be increased with the signal to noise ratio to obtain equal or better performance in case of header compression. This means that more robustness, corresponding to a longer time spent in the less compressed state, is needed. This behaviour can be explained by analysing the simulation logs, which show that when $\mathrm{L}$ is too low, the decompressor may be unable to create a static context, leading to relatively poor performance of the ROHC mechanism compared to the absence of header compression. This behaviour can be corrected by increasing L. These results show that, conversely to what usually reported in the literature, RoHC performance may degrade in case of long bursts and eventually badly attuned compression parameters, thus highlighting the importance of having a system simulator able to finely model high protocols, bit transmission and wireless channel as well.

In Fig. 5 we report an example of the application layer results. The PSNR achieved with the use of the Application Controller (here with full knowledge on the source stream) is compared to the non-adapted case for the Foreman ITU-T reference sequence (QCIF, $15 \mathrm{~Hz}, 17$ first GOPs) transmitted with jointly optimising H.264 AVC transmission and RTP FEC support. We can observe that the proposed optimisation performs better in general, apart for exceptional cases where unexpected channel realisations may by chance favour the non-adapted transmission. Even in such cases, only a limited time step is needed for the controller to adapt to the new CSI information, supposing that the channel coherence time is lower than the CSI feedback transmission delay.

Fig. 5 Received peak signal-to-noise ratio (PSNR) with and without adaptation

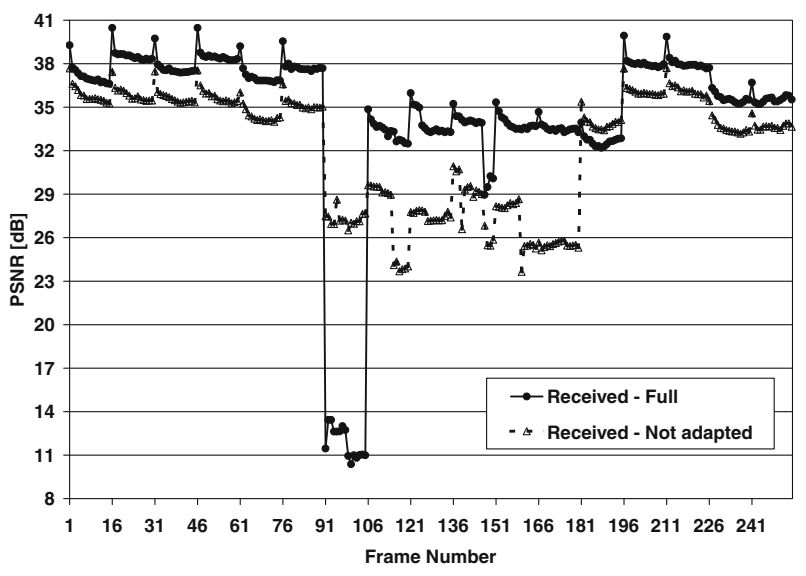


Finally, we present performance results for the proposed multi-user scheduling process. In particular, we have considered a single-input single-output (SISO) architecture, OFDM modulation with 256 sub-carriers and OFDMA/TDMA as multiple access schemes. The curves represent the overall throughput allocated by the BS Controller for different techniques, namely OFDMA with both WSR and SR maximization algorithms, as introduced in Section 4.4, and a simple TDMA technique based on a round-robin (RR) policy presented as a reference of more traditional transmission schemes. In all cases, adaptive modulation and coding (AMC) technique was used. In the simulations, the bandwidth available was fixed to $3.8 \mathrm{MHz}$ and the radio channel modelled as a Rayleigh block fading channel with block duration of $0.1 \mathrm{~s}$, with the addition of a log-normal fading with block duration of $2 \mathrm{~s}$ to take into account the effects of shadowing. With these radio layers conditions, we simulated the unicast transmission of multiple H.264-encoded Foreman sequences (CIF) to 5 users characterized by different median signal-tonoise ratio $E_{s} / N_{0}$, where $E_{s}$ represents the energy per OFDM symbol and $N_{0}$ is the one-sided power spectral density of the thermal noise. An additional user with non-real time (NRT) data traffic has been taken into account. The data throughput of each video sequence is around $520 \mathrm{kbps}$, while the NRT traffic is generated at a rate of $300 \mathrm{kbps}$. Figure 6 presents the corresponding results obtained running our complete simulation chain. Furthermore, Fig. 7 presents the PSNR achieved by the user with the lowest signal-to-noise ratio $\left(E s / N_{0}=25 \mathrm{~dB}\right)$ and either our OFDMA/WSR policy or TDMA/RR policy. Some decoded frames are also reported, as visual examples. The quality improvements achieved by our optimization techniques within the BS Controller is evident. Moreover, it is worth remarking here that, in case where the BS Controller is completely disabled, only 3 frames have been received and decoded by the considered user, while, in case our adaptive modulation and coding strategy is applied, 89 frames and 253 frames are received and correctly decoded with TDMA/RR and OFDMA/WSR, respectively.

In order to better demonstrate the interest of a complete simulation chain when compared to several easier-to-implement partial simulations, we report in Fig. 8

Fig. 6 Above Performance comparison of multi-user scheduling and bit allocation process, expressed as the overall number of bits encoded, modulated and transmitted over the radio channel obtained with the complete simulator. Below Average throughput achieved for the different users with the proposed techniques

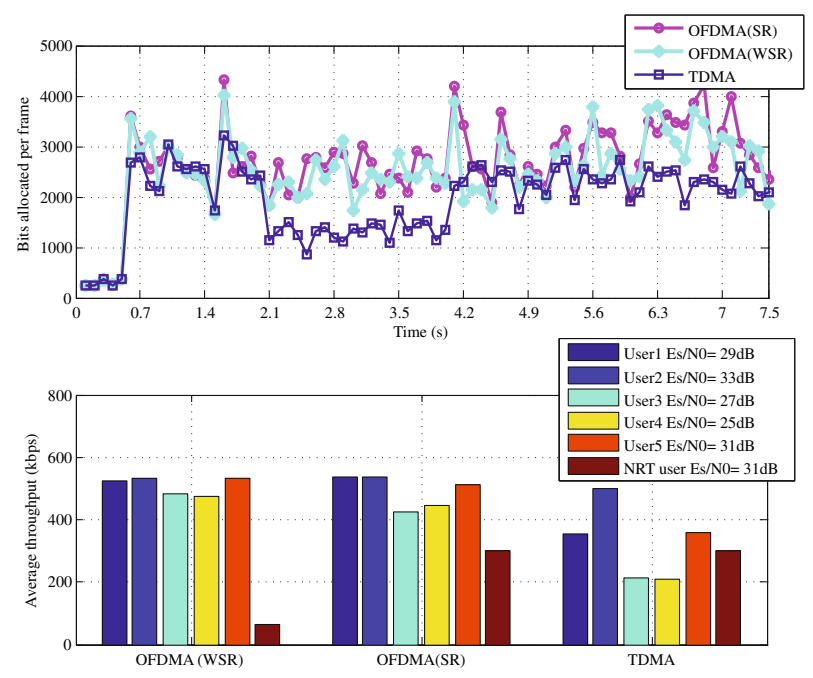




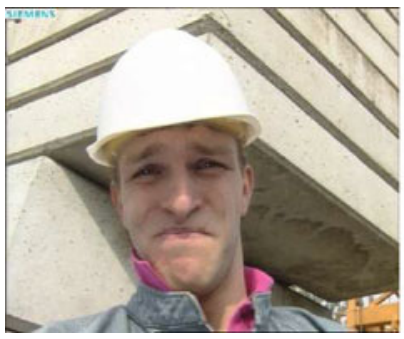

(a)

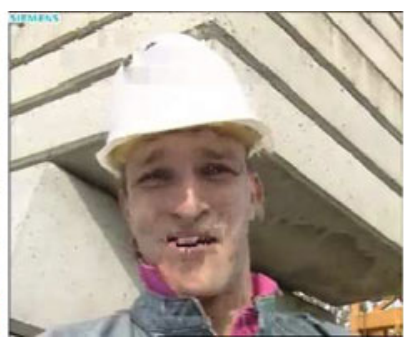

(b)

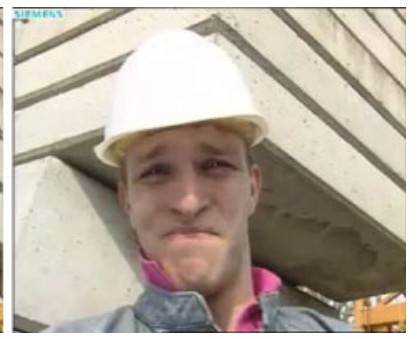

(c)

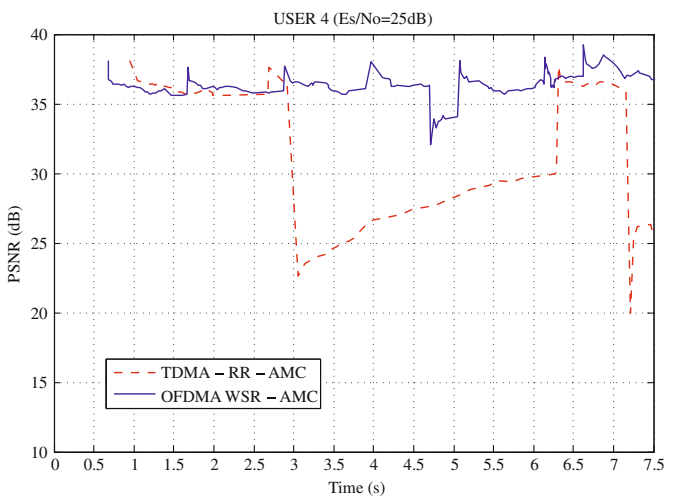

(d)

Fig. 7 a Original frame n. 40, before source encoding and transmission. b Frame $n .40$ received by user 4, with TDMA/RR policy. c Frame n. 40 received by user 4, with OFDMA/WSR policy. d User 4 experienced PSNR comparison between TDMA/RR and OFDMA/WSR techniques
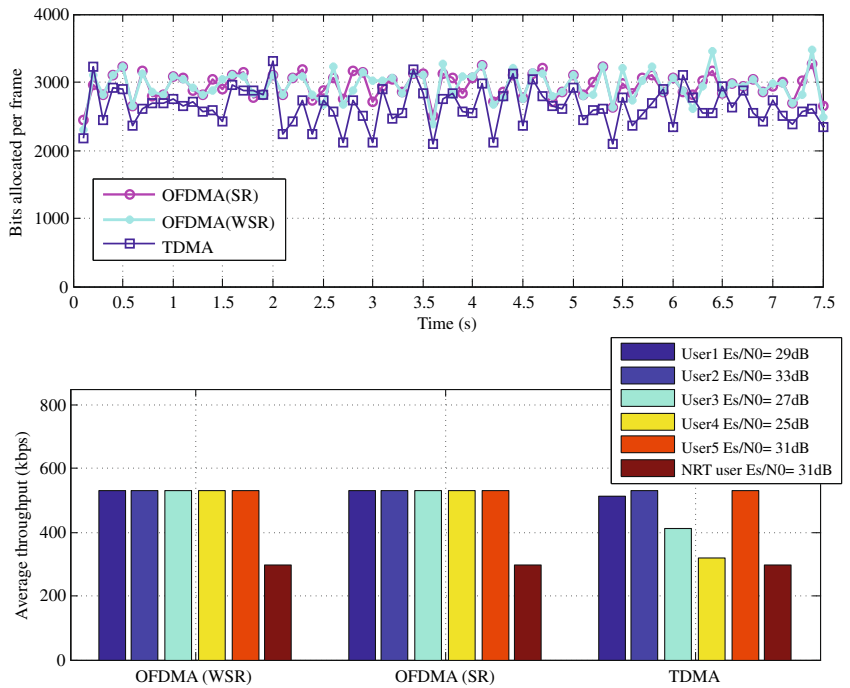

Fig. 8 Above Performance comparison of multi-user scheduling and bit allocation process, expressed as the overall number of bits that can be transmitted over the radio channel, obtained executing the scheduling and allocation modules separately from the rest of the chain. Below Average throughput achieved for the different users with the proposed techniques 
results obtained for the multi-user scheduling and radio resource allocation when the correspondent modules (included in the BS Controller in the OPTIMIX simulator) are run independently. Obtained with the same radio channel conditions as those used for Fig. 6, results obtained with the partial simulation are shown to tend to be too optimistic and less accurate. This is due to the fact that all the main elements of a real communication chain have not been taken into account, i.e. the real video packets, IPv6 network, channel coding and modulation at the PHY layer, etc. as well as specific cross-layer strategies such as our AMC approach. On the contrary, the end-to-end simulation tool allows to develop and test cross-layer optimizing techniques in more realistic situations. Typically, it can be noted from Fig. 6 that the proposed WSR maximization algorithm applied in the OFDMA scenario guarantees high throughput to all the high-priority real-time users, leaving the remaining bandwidth to the low-priority NRT data. The SR maximization, on the contrary, tends to favour the users with high $E_{s} / N_{0}$, which leads in the considered situation users 3 and 4 not to receive sufficient data rates.

\section{Conclusions}

The OPTIMIX project main goal is to effectively exploit the available bandwidth on wireless links such as $802.11 \mathrm{a} / \mathrm{g} / \mathrm{n}$ ones, in the specific context of point to multi-points multimedia streaming. The complexity of the complete transmission chain, when one considers the number of parameters that can (or not) be jointly optimised, explains the interest and the necessity of establishing a reference simulation chain, shared by all the project partners in order to allow the optimisation of the different aspects of the multimedia transmission in a joint manner. Ultimately indeed, the goal is to establish efficient rules for increasing the perceived quality of service for the enduser(s) and recommend accordingly implementation of our controlling strategies in real networks. First results have already been obtained with the OPTIMIX simulation chain, allowing to validate the information control exchange, a new multiuser scheduling technique, and an original joint source/application FEC solution as well as to demonstrate the interest of a high fidelity simulation. Knowing the main parameters that really influence the perceived quality of service and how to jointly attune them is the ultimate objective of OPTIMIX, and the reason why this global simulation framework has been set-up. Developed over OMNeT++, the framework simulator is also expected to allow to prove the interest of the controlling algorithms with respect to separate coding procedures, and provide representative figures in terms of perceived quality for the end-users.

Acknowledgements The authors would like to thank their colleagues, who have participated in IST PHOENIX and ICT OPTIMIX projects. In particular, is gladly recognized the help of our engineer and intern colleagues who have participated to the difficult task of putting all parts of the simulation framework together, with special thanks to Prof. Marco Chiani and Prof. Lajos Hanzo as well as Benjamin Gadat and Alessandra Tripodi. Further information on the OPTIMIX project can be found on http://www.ict-optimix.eu. 


\section{References}

1. 3GPP TS 26.346 V7.4.0 (2007) Technical specification group services and system aspects: multimedia broadcast/multicast service; protocol and codecs

2. Bajic IV (2007) Efficient cross-layer error control for wireless video multicast. IEEE Trans Broadcast 53(1):276-285

3. Baugher $M$ et al (2004) The Secure Real-Time Transport Protocol (SRTP). In: IETF RFC 3711

4. Bernstein DJ (2008) ChaCha, a variant of Salsa20. http://cr.yp.to/papers.html\#chacha. Accessed January 2008

5. Bormann C et al (2001) Robust Header Compression (ROHC): framework and four profiles: RTP, UDP, EPS, and uncompressed. In: IETF RFC 3095

6. Casner S et al (2003) RTP: a transport protocol for real-time applications. In: IETF RFC 3550

7. Deering $S$ et al (1998) Internet protocol, version 6 (IPv6) specification. In: IETF RFC 2460

8. Fu F, van der Schaar M (2009) Cross-layer optimization with complete and incomplete knowledge for delay-sensitive applications. In: Proc. of IEEE packet video workshop, pp 1-10

9. Hagenauer J, Stockhammer T (1999) Channel coding and transmission aspects for wireless multimedia. Proc IEEE 87(10):1764-1777

10. Hsu J-L, van der Schaar M (2009) Cross-layer design and analysis of multiuser wireless video streaming over 802.11e EDCA. IEEE Signal Process Lett 16(4):268-271

11. H.264 joint verification model version 12.1. http://iphome.hhi.de/suehring/tml. Accessed March 2007

12. Kasch W, Ward J, Andrusenko J (2009) Wireless network modeling and simulation tools for designers and developers. IEEE Commun Mag 47(3):120-127

13. Kawadia V, Kumar PR (2005) A cautionary perspective on crosslayer design. IEEE Wirel Commun 12(1):3-11

14. Kohler E et al (2006) Datagram Congestion Control Protocol (DCCP). In: IETF RFC 4340

15. Kopke A et al (2008) Simulating wireless and mobile networks in omnet++ the mixim vision. In: SIMUTools

16. Liu Z, Liu H, Wang Y (2006) Cross layer adaptation for H.264 video multicasting over wireless LAN. In: Proc. of IEEE ICME'06, pp 1121-1124

17. Mäkelä J, Pentikousis K (2007) Trigger management mechanisms. In: Symposium ISWPC'07, pp 378-383. San Juan, Puerto Rico

18. Martini MG, Mazzotti M, Lamy-Bergot C, Huusko J, Amon P (2007) Content adaptive network aware joint optimization of wireless video transmission. IEEE Commun Mag 45(1): 84-90

19. Mobility framework (MF) for simulating wireless and mobile networks using OMNeT++. http://mobility-fw.sourceforge.net/. Accessed March 2008

20. Moskowitz R, Nikander P, Jokela P, HendersonT (2008) Host identity protocol. In: IETF RFC 5201

21. OMNeT++. Discreet event simulation system. http://www.omnetpp.org. Accessed March 2008

22. OPTIMIX project team (2010) OMNeT++ based OPTIMIX system simulator. http://www.ictoptimix.eu/index.php/OPTIMIXsim. Accessed April 2010

23. Postel J (1980) User Datagram Protocol (DCCP). In: IETF RFC 768

24. Schulzrinne H et al (1998) Real Time Streaming Protocol (RTSP). In: IETF RFC 2326

25. Shannon CE (1948) A mathematical theory of communication. Bell Syst Techn J 27:379-423, 623-656

26. Srivastava V, Motani M (2005) Cross-layer design: a survey and the road ahead. IEEE Commun Mag 43(12):112-119

27. Timmerer $\mathrm{C}$ et al (2008) An integrated management supervisor for end-to-end management of heterogeneous contents, networks, and terminals enabling Quality of Service. In: EUMOB'08

28. Uitto M, Vehkapera J (2009) Spatial enhancement layer utilisation for SVC in base layer error concealment. In: MOBIMEDIA'09, London, UK

29. Lacage M, Henderson $T$ (2006) Yet another network simulator. Institut National de Recherche en informatique et Automatique (INRIA). http://hal.inria.fr/inria-00078318/fr/. Accessed July 2006

30. Zahariadis Th, Negru O, lvarez F (2008) Scalable content delivery over P2P convergent networks. In: IEEE ISCE'08, Vilamoura, Portugal 


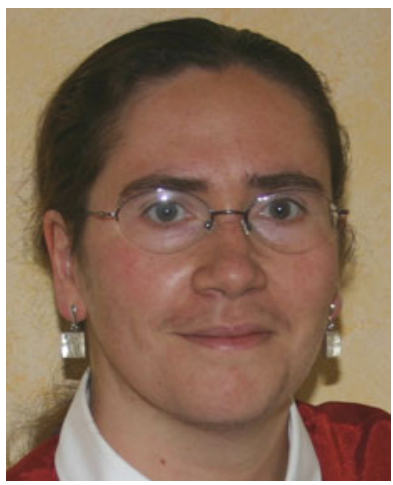

Catherine Lamy-Bergot received the electrical engineering degree from the École Nationale Supérieure des Télécommunications (E.N.S.T.), Paris, France, in 1996, the Diplôme d'Études Approfondies (D.E.A.) in Telecommunications also in 1996 and the Ph.D. degree from the E.N.S.T. in 2000 with a thesis on high spectral efficiency communications over fading channels. She was with Philips Research France from 2000 to 2002 where she worked on joint source and channel coding techniques and participated to the European project JOCO. Since Sept. 2002, she is with THALES Communications as a Senior Scientist in digital communications. She participated to different French National Research projects and European projects both as technical manager and researcher, among which IST project PHOENIX, Celtic project BOSS and ICT project OPTIMIX. She is now System Architect for Aero and Naval communications, in charge of reflections for new solutions of networked wireless radio-communications.

Dr. Lamy-Bergot is Senior IEEE Member since 2006. Her technical fields of interest include system design and architecture optimisation, HF communications, multimedia applications, quality of service strategies, iterative decoding techniques, high efficiency modulations, error correction codes, unequal error protection techniques, robust header compression, soft output decoding, joint source channel coding techniques and end-to-end wireless communication optimisation.

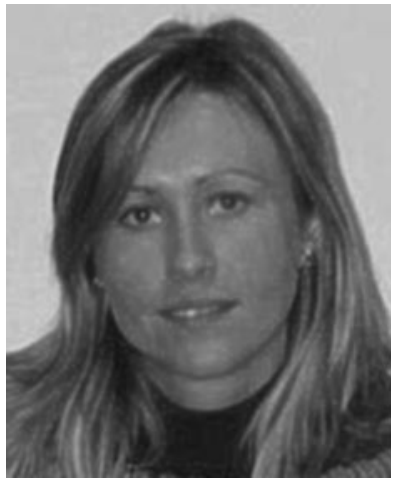

Roberta Fracchia received her master degree in Telecommunications Engineering from Politecnico di Torino, Italy, in 2002 and her PhD from Politecnico di Torino, Italy, in 2005. In 2003 and 2004 she was a visiting researcher in the Information Systems Networking Lab of Prof. Balaji Prabhakar at the Electrical Engineering Department of Stanford University, CA, USA. In 2002, and between 2004 and 2006 she had a research contract with CNIT (Italian National Inter-university Consortium for Telecommunications). In 2006 she joined Motorola Labs in Saclay, Paris, France, where she worked as Senior Research Engineer. She participated to the ICT projects WINNER2 and E3. She actively 
contributed to IEEE standardization of $802.11,802.16$ and P1900. She was Motorola representative at IEEE $802.11 \mathrm{n} / \mathrm{ac}$ meetings and she participated to the project definition (PAR) of IEEE802.11ac. Since November 2008 she joined THALES Communications as a Senior Scientist in signal processing and networking. She is currently leading ICT project OPTIMIX and the RNRT national project DITEMOI.

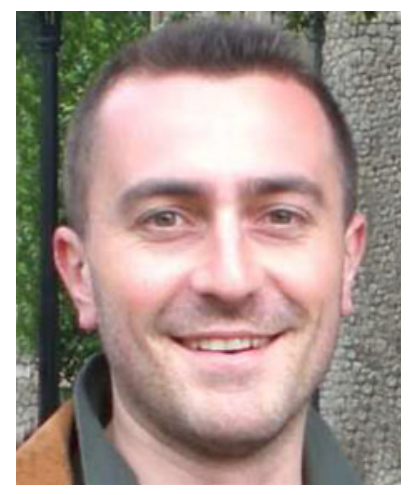

Matteo Mazzotti received the degree in Telecommunication Engineering (with the highest honors) and the Ph.D. degree in Electronic, Computer Science and Telecommunication Engineering in July 2002 and May 2007, respectively, from the University of Bologna, Italy. Now he is a postdoctoral researcher with CNIT/DEIS, University of Bologna. His main research interests cover digital signal processing techniques, multimedia communications, source and channel coding, and multiuser wireless communication systems.

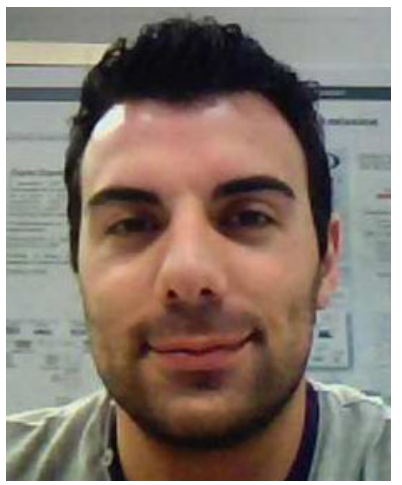

Simone Moretti was born in Rimini, Italy, on 29 June 1983. He received the degree in Electronic and Telecomunications Engineering in July 2009 from the II Facoltà di Ingegneria Cesena, University of Bologna. Now he works as a project collaborator with the Consorzio Nazionale Interuniversitario per le Telecomunicazioni (CNIT). His main research interest cover radio resource optimization, multiuser scheduling and wireless communications systems. 


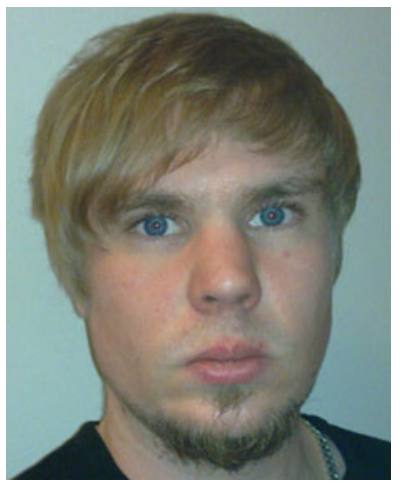

Esa Piri is working as a Research Scientist at VTT Technical Research Centre of Finland. He received his M.Sc. (Tech.) from the University of Oulu, Finland, in 2008. His research interests and activities are mainly related to seamless networking and mobility management issues in heterogeneous networks.

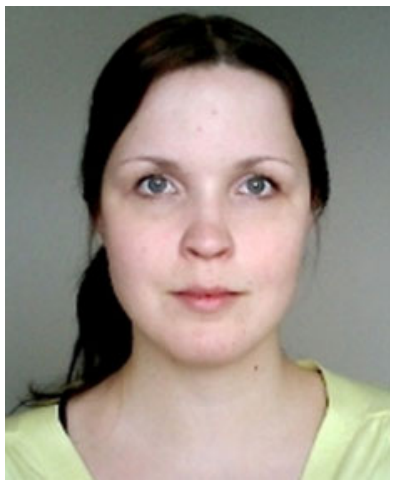

Tiia Sutinen has a M.Sc. (Tech.) degree that she received from University of Oulu, Finland, in 2004. Currently, she is working as a research scientist and a project manager at VTT Technical Research Centre of Finland. She has gained experience of several years in national and international research projects. The main focus in her research work is in cross-layer assisted adaptive transmission of scalable video in heterogeneous multi-access networks. In specific, her interests lie in applicationaware mobility management and application adaptation in different network scenarios, including different types of handovers and link-level mechanisms to improve video delivery over wireless links. Under these topics, she is pursuing a Ph.D. degree and has also published her work in international scientific forums. 


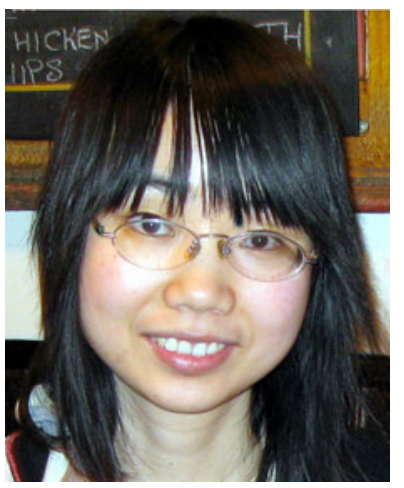

Jing Zuo received her bachelor and master degree in Communication Engineering from Jilin University, P.R.China, in 2006 and 2008, respectively, and now is a PhD student in Communications Research Group, School of Electronics and Computer Science in University of Southampton. Since March 2009, she has been part of a team working on the OPTIMIX European project. She is a member of the IEEE and her research interests are mainly in channel coding and modulation, cooperative communication, routing algorithm in Ad hoc network, and cross-layer optimization in wireless network. She has published a number of papers in these areas

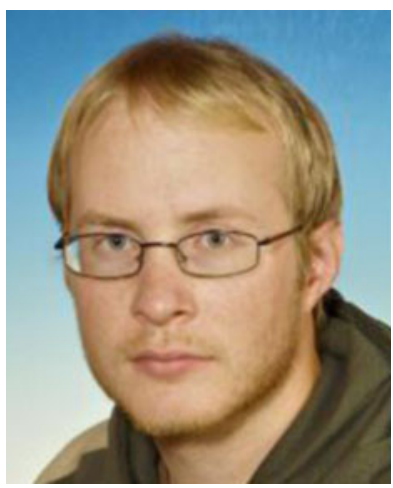

Janne Vehkaperä received the M.Sc. (Tech.) in electrical engineering in 2004 from the University of Oulu, Finland. He joined VTT Technical Research Centre of Finland, Oulu, in 2002, where he is currently working as a research scientist and project manager in the field of multimedia communication. He is currently part of a research team focused on multimedia communication which is part of Media Technologies knowledge centre. His main research interests include crosslayer aspects of video transmission, scalable video coding and video error resilience. 


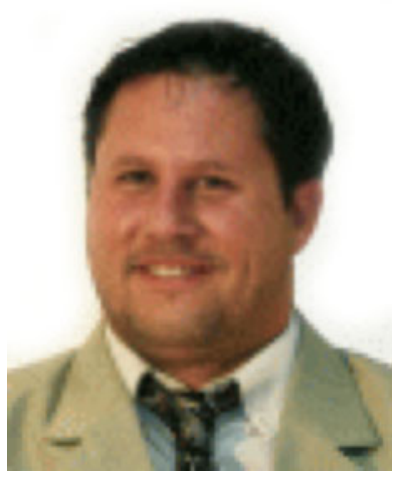

Gabor Feher graduated in 1998 as a informatics engineer at the Budapest University of Technology and Economics. He received his Ph.D. degree in 2004 in the field of resource control in IP networks. Currently he is an associate professor at the Department of Telecommunications and Media Informatics. He gives lectures in the topics of network and multimedia security. Since 1997 he is a member of the High Speed Network Laboratory of the Department and is involved in several national and international research projects.

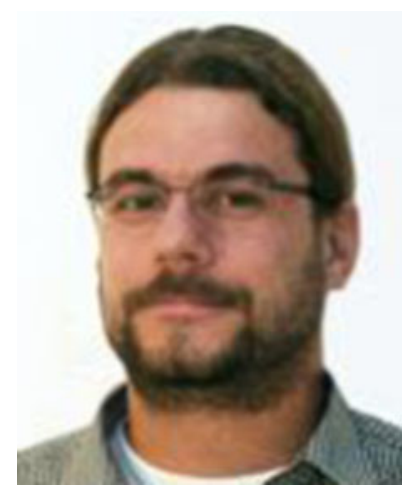

Gabor Jeney received his M.Sc. degree in 1998 from the Technical University of Budapest in the field of Mobile and Optical Communications, and the Ph.D. degree from the same university. Since 1999 he has been a member of the IEEE and HTE. He has been recently involved in several European projects (e.g. IST-Phoenix, IST-Anemone, ICT-Optimix, and Celtic-BOSS). His main research interests cover mobile communication systems, neural networks, advanced signal reception techniques, the mobility support of IPv6, etc. 


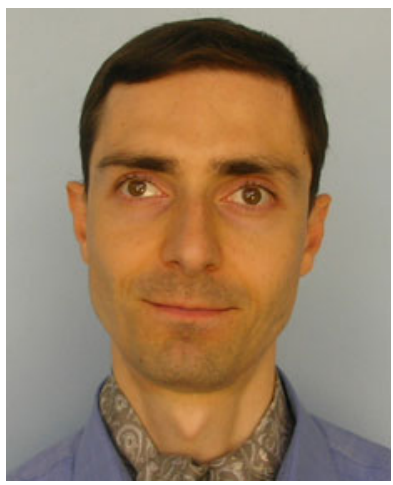

Gianmarco Panza received the degree in Information Science Engineering at University of Padova, Italy in 1998 and a Master degree in ICT at CEFRIEL/Politecnico di Milan, Italy in 1998. He started his working career as a researcher for ALCATEL ITALIA, Italy in the field of Quality of Service over IP networks in 1998. Since 1999, he has worked as a senior researcher and consultant on network systems at CEFRIEL Milan, Italy. Aside activities have included teaching in ICT master courses and providing training to private company employees, about Quality of Service over IP networks, Voice over IP and MPLS (Multi-Protocol Label Switching).

He managed and participated to several EU IST projects (e.g. BASS, MOICANE, JOCO, PHOENIX, Membrane, MULTINET, OPTIMIX), as well as a wide variety of national and international projects regarding all the aspects of network and service provisioning in LAN, MAN and WAN environments, from specification to simulation modelling, realization and testing (e.g. about the first European satellite network, the Pirelli skyscraper indoor telecommunication infrastructure, advanced services over power-line networks, the contribution and distribution networks of media operators, the assessment of FP5 EU project in the broadband domain, innovative network solutions to eliminate the digital divide).

He had several IEEE publications in Digital Signal Processing and Networking areas and became senior IEEE Member in 2008.

He also achieved the PMP certification in project management by PMI (Project Management Institute) in 2009.

His interests and expertise are in Quality of Service in IP networks, Voice over IP, MPLS, multimedia systems, simulation modelling, and network planning, design, testing and assessment.

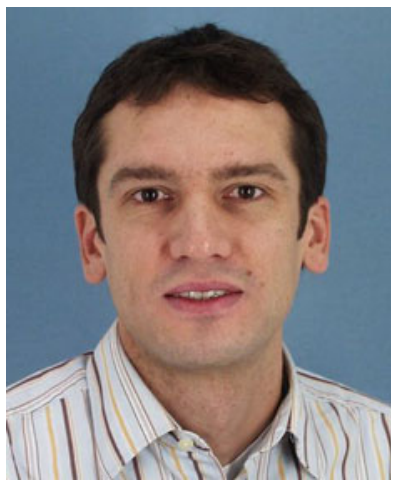

Peter Amon received his Dipl.-Ing. degree in electrical engineering from the University of ErlangenNuremberg, Germany in 2001, where he specialized in communications and signal processing. In 2001 
he joined Siemens Corporate Technology, Information and Communications division, where he is currently working as a research scientist in the Real-time Vision and Industrial Imaging department. In this position, he is and has been responsible for several research projects. His research field encompasses video coding, video transmission, error resilience, and joint source channel coding. In that area, he has authored or co-authored several conference and journal papers, and holds several patents. He also actively contributed to the standardization bodies ITU-T VCEG and ISO/IEC MPEG in the field of video coding and related topics. 